Rückfluß erhitzt. Dabei entsteht mit $80 \%$ Ausbeute Tetra-(dimethylamino)-äthylen (2), das man durch Destillation reinigt.

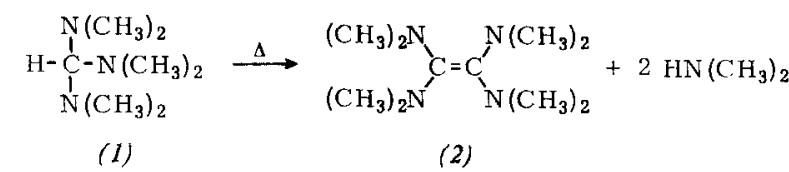

Die Verbindung (2) wurde erstmals von Pruett et al. aus Trifluorchloräthylen mit Dimethylamin synthetisiert (Ausbeute

$54 \%{ }^{[2]}$. Sie entsteht auch bei der thermischen Zersetzung von Aminalestern [3]. Die für dieses Verfahren angegebenen Ausbeuten sind nur gering.

Eingegangen am 15. August $1966 \quad$ [Z 311]

[1] H. Bredereck, F. Effenberger u. Th. Brendle, Angew. Chem. 78, 147 (1966); Angew. Chem. internat. Edit. 5, 132 (1966).

[2] R. L. Pruett et al., J. Amer. chem. Soc. 72, 3646 (1950).

[3] H. E. Winberg et al., J. Amer. chem. Soc. 87, 2055 (1965); US-Pat. 3239534 (8. März 1966).

\title{
Eine neue Synthese \\ von Tetra-(dimethylamino)-äthylen
}

Von Prof. Dr. H. Bredereck, Dr. F. Effenberger und cand. chem. H. J. Bredereck

Institut für Organische Chemie,

Technische Hochschule Stuttgart

Trisdimethylaminomethan (1) konnten wir aus Tetramethylformamidiniumsalzen mit Alkalimetalldimethylamiden darstellen [1]. Wir haben nun gefunden, daß (1) leicht Dimethylamin abspaltet, wenn man es unter Stickstoff 2 Std. unter 